\title{
Synergistic evolutionary model for dynamic evaluation of energy saving and emission reduction in thermal power enterprise
}

\author{
Zhang Lei ${ }^{1,2}$, Li Na-na ${ }^{1}$, Zhao Hui-ru' ${ }^{1}$, Yang Kun ${ }^{1}$ \\ ${ }^{1}$ The Institute of Economics and Management, North China Electric Power University, Beijing, China \\ ${ }^{2}$ State Development and Investment Corporation, Beijing, China \\ Email address: \\ nancyli1007@163.com (Li Na-na)
}

\section{To cite this article:}

Zhang Lei, Li Na-na, Zhao Hui-ru, Yang Kun. Synergistic Evolutionary Model for Dynamic Evaluation of Energy Saving and Emission Reduction in Thermal Power Enterprise. International Journal of Energy and Power Engineering. Vol. 3, No. 2, 2014 , pp. 46-51. doi: $10.11648 /$ j.ijepe.20140302.12

\begin{abstract}
Through analyzing the complexity of energy saving and emission reduction system in thermal power enterprise, we can find that this system affected by various elements within the system, policies and technologies in the external environment. Therefore, in order to evaluate the performance of energy saving and emission reduction in thermal power plants, both the complex nonlinear relationships among internal elements and the environmental impact should be considered. The state variables of system were determined based on collaborative learning theory, meanwhile a synergistic evolutionary model for dynamic evaluation of energy saving and emission reduction in thermal power enterprise was proposed. Based on the actual data of a thermal plant, the empirical results showed that the grid electricity and soot emissions of per kwh are the order parameters in this system, which plays a key role on the evolution of the energy saving and emission reduction system. Furthermore, the order parameters are the foundation of the performance evaluation.
\end{abstract}

Keywords: Thermal Power Enterprise, Energy Saving and Emission Reduction, Dynamic Evaluation, Evolutionary Model, Order Parameter

\section{Introduction}

Faced with the severe pressure of energy and environment, thermal power plants as the major industry of China's energy generation and consumption need to assume more responsibility. In recent years, thermal power enterprises have been strengthening energy saving and emission reduction management continuously, increasing investment of technology innovation, so as to improve their performance of energy saving and emission reduction [1-3].

The thermal power plant is a dynamic system, so the performance evaluation on its energy saving and emission reduction should be on the perspective of dynamic development of entire system, and the dynamic relationship between the various elements within the system should be explained. The thermal power is an internal complex system constituted by the combustion system, steam systems, power generation systems, appropriate auxiliary equipment and external environment. In the production process of thermal power plant, different status of electricity demand, the natural condition and policy situation will affect the operation efficiency of equipment as well as the energy consumption and pollutant emission level. In dynamic development of thermal power plant, there exists interaction relations between each element, meanwhile the system is also affected by the external environment such as fuel supply, load level, government restrictions and so on. Therefore, the synergetic theory of complex system science can be used to evaluate the performance of energy saving and emission reduction in thermal power enterprises. Under a given external conditions, the analysis of interaction between various elements is proposed to identify the key factors which affect the energy saving and emission reduction performance in thermal power enterprise, and the dynamically evaluation can be obtained by studying the changes of these key factors. 


\section{Synergetic Theory and Synergetic Characteristics of Energy Saving and Emission Reduction in Thermal Power Plant}

Synergetic theory which is put forward by the German physicist Haken, mainly research the complex system composed of interrelated factors. The theory proposes that the competition and collaboration relationship between internal variables continuously promote the system evolution, while the property and role of different variables are unequal in the system evolution [4]. When the system is in the critical point, some variables change rapidly while some change slowly. The slow variable is the key variable that dominates the eventually structure of system and the order degree of system feature, which is called the order parameter. The order parameter will change with the system situation, so the evolution of the system operation states can be explored by the order parameter. Therefore, it is important to define the order parameter in the dynamic performance evaluation of the energy saving and emission reduction of thermal power enterprise [5,6]. Before researching the system status based on the synergetic theory, we should analyze whether the system has the general characteristics of synergetic theory. In this paper, the energy saving and emission reduction in thermal power plant is be provided with the open, non-equilibrium, and synergistic characters.

When there is no substance, energy and information exchange, the system will eventually evolve into a disordered situation, which is based on the dissipative structure theory. In the production process of thermal power plant, the operation efficiency of system will be affected by electricity load, coal and natural conditions. The higher the load rate is, the higher the unit efficiency, coal consumption rate, power consumption rate and efficiency of stock desulfurization device are. Meanwhile, the energy saving and emission efficiency of the plant will affect the natural environment and the intensity of related policies. Therefore, the substance, energy, and information of nature and social environment have a continuous impact on the operation of the system, while the system is constantly transporting material, ability and information to the outside world. So the energy saving and emission reduction in thermal power plants has an open feature. It is the open features that energy saving and emission reduction of thermal power enterprises can develop to an orderly state.

Dissipative structure theory suggests that the balance structure is a "dead" structure. The open systems can develop into an orderly situation only in the non-equilibrium state $[7,8]$. In the production process of thermal power plants, the system is constantly exchanging substances and information with the outside world. Since the external conditions such as electricity load, coal and natural conditions are constantly changing, the operating status is also affected with a non-equilibrium dynamics state. The non-equilibrium feature of energy saving and emission reduction in the thermal power plant makes the system evolve towards an orderly direction.

Synergetic theory reveals an evolution process of the system, that is from the "old structure" to "instability structure" to a "new structure". Similarly, the energy saving and emission reduction of thermal power plant is experiencing the evolution from old structure to a new structure, with a synergistic property [9]. From the investment to operation, the devices in the thermal power plant continuously adjusted its running ways to suit different conditions. The plant continuously breaks the inefficiently old structure, and evolves to a new and efficient structure. And the operation efficiency, coal consumption rate, power consumption and pollutant emission levels can be improved. This is due to the existence of competition and synergy between the various devices within the system, and it makes the variables complement each other, which leads to a better operating result. Therefore, the energy saving and emission reduction in thermal power company has a synergistic feature.

\section{Establishing the Co-Evolution Model for Dynamic Performance Evaluation of Energy Saving and Emission Reduction in Thermal Plant}

\subsection{Construction of Co-Evolution Model}

Through building the co-evolution model for the system, the synergetic subject can study the evolution regular pattern of system and explore the key variable, constant, and the linkage among the variables. The basic evolution equation of synergetic subject can be generally written in the form of Langevin's equation as follows:

$$
\frac{d q}{d t}=K(q, a)+f(t)
$$

where, $\frac{d q}{d t}$ represents the rate of change of different state variables; $q$ is the state variable; $\alpha$ is the control parameter; $f(t)$ is the random fluctuating force. The evolution model contains of $q$ and the different order derivative differential equations of $q$.

Energy saving and emission reduction performance indicators of thermal power plant are generally non-consecutive monthly data. The Gray theoretical model can deal with discrete data sequence well. It also can reveal the changing laws and characterize the evolution and development state of data ${ }^{[10]}$. Therefore, co-evolution model of energy saving and emission reduction of thermal power plant can be constructed through combining the gray theoretic model and synergetic subject. The traditional $G M(1, N)$ model is defined as follow: 


$$
\frac{d x_{i}}{d t}+a_{i} x_{i}=\sum_{j \neq i}^{N} b_{j} x_{j}
$$

where, $-a_{i} x_{i}$ is the developing term reflecting the growth of the variables in evolution process; $b_{j} x_{j}$ is the driven term reflecting the driving effect of the other variables to variable $i$, and $\mathrm{N}$ is the amount of variables.

According to the synergetic subject, the change rate of variables is limited by their own development capacity and affected by the interaction among variables and external interference (fluctuation) in the evolution process of system. Among them, the self-development capacity is reflected by the developing terms of the variable; interaction among variables is the net effect of competition and coordination role between variables, that is difference between hinder capability and drive capability; random fluctuations is primarily related to random factors. Learn from the literature[11,13], $a_{i} x_{i}(t)$ is the self-developing capability of variable $\mathrm{i}$ at time $\mathrm{t}, a_{i}$ is the damping coefficient of the variable $\mathrm{i}$, reflecting the decay rate of the $\mathrm{i}$ indicator; $b_{j} x_{j}(t)$ is the driving force of the rest sub-variables on variable $\mathrm{i}, c_{j}\left(x_{j}\right)^{2}(t)$ is the hindering factor, where $b_{j}$ is the driving coefficient, $c_{j}$ is the obstruction coefficient and $f_{i}(t)$ is outside interference. The evolution equation of the system can be generally written in the form of Langevin's equation:

$$
\frac{d x_{i}}{d t}+a_{i} x_{i}=\sum_{j \neq i}^{m}\left[b_{j} x_{j}+c_{j}\left(x_{j}\right)^{2}\right]+f_{i}(t)
$$

Where, $m$ represents the number of variables. For any nonlinear evolution system, internal factors are the essential reason for system evolution, external factors are random reasons contributed to the occurrence of a qualitative change in the critical point for the system evolution. In this paper, the deviation of order parameter caused by internal factors is mainly analyzed. In the evolution process of energy saving and emission reduction of thermal power plant, the $f_{i}(t)$ is the outside interference[14]. This article mainly analyzed the internal cause that makes the order parameter deviate.

For any complex nonlinear system, the nature factors of system evolution are the internal factors and the external factors are merely random factors at the critical point. When the residuals of system has a zero mean and one variance, random fluctuations of the system can be regarded as no effect, and the internal factors are the critical factors in the system evolution. So the changes of order parameters are mainly analyzed in this paper [15].

\subsection{Solving the Model}

(1) Dimensionless processing

Since there are different dimensions of the original data, the variable date need to be processed by dimensionless way. Assuming that the original sequence and the processed sequence of state variable $i$ respectively are $x_{i}(t)$ and $x_{i}^{(0)}(t)$. The processed sequence is $x_{i}^{(0)}(t)=\frac{x_{i}(t)-x_{i}\left(t_{b}\right)}{x_{i}\left(t_{a}\right)-x_{i}\left(t_{b}\right)}$, in which, $x_{i}^{(0)}\left(t_{a}\right)=\max _{t=1,2, \cdots n} x_{i}(t)=1, x_{i}^{(0)}\left(t_{b}\right)=\min _{t=1,2, \cdots n} x_{i}(t)=0$, and $n$ is the number of samples.

(2) Data accumulation

According to the principle of Grey theory, the original sequences' variation is not obvious, and there will be an obviously exponential growth law when they are processed by accumulation. Thus, before solve the model, the data accumulation should be progressed. The accumulation sequence is $x_{i}^{(1)}(t)$, and $x_{i}^{(1)}(t)=\sum_{j=1}^{t} x_{j}^{(0)}(t)$.

(3) solving the parameters

Assuming that $f_{i}(t)=0,-a_{i}=b_{i i}$, then the evolution equations of system are:

$$
\left\{\begin{aligned}
\frac{d x_{1}^{(1)}}{d t} & =\sum_{j=1}^{m} b_{1 j} x_{j}^{(1)}+\sum_{j \neq 1}^{m} c_{1 j}\left(x_{j}^{(1)}\right)^{2} \\
\frac{d x_{2}^{(1)}}{d t} & =\sum_{j=1}^{m} b_{2 j} x_{j}^{(1)}+\sum_{j \neq 2}^{m} c_{2 j}\left(x_{j}^{(1)}\right)^{2} \\
\frac{d x_{3}^{(1)}}{d t} & =\sum_{j=1}^{m} b_{3 j} x_{j}^{(1)}+\sum_{j \neq 3}^{m} c_{3 j}\left(x_{j}^{(1)}\right)^{2} \\
& \vdots \\
\frac{d x_{m}^{(1)}}{d t} & =\sum_{j=1}^{m} b_{m j} x_{j}^{(1)}+\sum_{j \neq m}^{m} c_{m j}\left(x_{j}^{(1)}\right)^{2}
\end{aligned}\right.
$$

Discretization processing the change rates of the equation of the left side, $\frac{d x_{i}^{(1)}}{d t}=x_{i}^{(1)}(t)-x_{i}^{(1)}(t-1)=x_{i}^{(0)}(t), t=2,3, \cdots n$, then:

$$
x_{i}^{(0)}(t)=\sum_{j=1}^{m} b_{i j} x_{j}^{(1)}(t)+\sum_{j \neq i}^{m} c_{i j}\left(x_{j}^{(1)}(t)\right)^{2}
$$

Respectively assuming $t=2,3, \cdots n$ in equation (4), then when $i=1$, the equations are as follows:

$$
\left\{\begin{array}{c}
x_{1}^{(0)}(2)=\sum_{j=1}^{m} b_{1 j} x_{j}^{(1)}(2)+\sum_{j \neq 1}^{m} c_{1 j}\left(x_{j}^{(1)}(2)\right)^{2} \\
x_{1}^{(0)}(3)=\sum_{j=1}^{m} b_{1 j} x_{j}^{(1)}(3)+\sum_{j \neq 1}^{m} c_{1 j}\left(x_{j}^{(1)}(3)\right)^{2} \\
\vdots \\
x_{1}^{(0)}(n)=\sum_{j=1}^{m} b_{1 j} x_{j}^{(1)}(n)+\sum_{j \neq 1}^{m} c_{1 j}\left(x_{j}^{(1)}(n)\right)^{2}
\end{array}\right.
$$

Representing equation (6) as a matrix form- $y=X \beta$ :

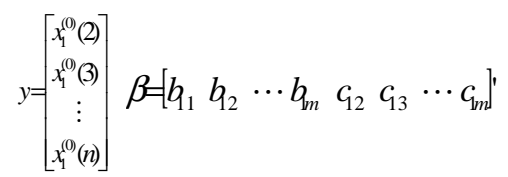

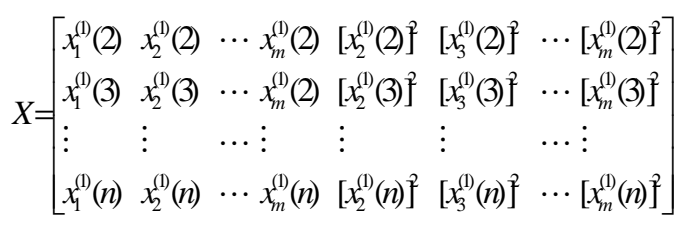

Estimate the parameters of the multiple linear regression 
model as equation (6), then the expression equation of coefficient matrix- $\beta$ can be obtained. Similarly, calculating the evolution equation of different state variables when $i=2,3, \cdots, m$, the co-evolution equations of the system can be received.

\section{Empirical Analysis}

Some variables which indicate the performance of energy saving and emission reduction respectively were selected as the state variables in dynamic co-evolution model of energy saving and emission reduction in thermal power plants. They are coal consumption, power consumption rate, water consumption per unit power, soot emissions per unit power, $\mathrm{SO} 2$ emissions per unit power and nitrogen oxide emissions per unit power six state variables. A $2 \times 30 \mathrm{MW}$ thermal power plant was selected as the empirical analysis object. This plant responded the call of the nation positively, it promoted the innovation and application of energy saving and emission reduction technology $[16,17]$. The state variables data is from the statistical data between 2009 to the second quarter of 2013. Based on the above method to build co-evolution model on the dynamic energy saving and emission reduction performance evaluation, and the stepwise regression analysis in the Eviews 6.0 software is used to fit. The final equation can used to determine the order parameter of the energy saving and emission reduction in thermal power plant.

Table 1. Actual data of state variables in energy saving and emission reduction system of thermal power plant.

\begin{tabular}{|c|c|c|c|c|c|c|}
\hline Time & $\begin{array}{l}\text { Coal } \\
\text { consumption } \\
(\mathrm{g} / \mathrm{kwh}) \\
\end{array}$ & $\begin{array}{l}\text { Power } \\
\text { consumption } \\
\text { rate }(\%) \\
\end{array}$ & $\begin{array}{l}\text { Water consumption } \\
\text { per unit power } \\
(\mathrm{g} / \mathrm{kwh})\end{array}$ & $\begin{array}{l}\text { soot emissions } \\
\text { per unit power } \\
(\mathrm{g} / \mathrm{kwh})\end{array}$ & $\begin{array}{l}\text { SO2 emissions per } \\
\text { unit power } \\
(\mathrm{g} / \mathrm{kwh})\end{array}$ & $\begin{array}{l}\text { nitrogen oxide } \\
\text { emissions per unit } \\
\text { power }(\mathrm{g} / \mathrm{kwh})\end{array}$ \\
\hline 2009 1st Quarter & 354.3449 & 8.6178 & 1.3107 & 0.0744 & 0.7529 & 1.7012 \\
\hline 2009 2st Quarter & 352.7797 & 8.3515 & 0.5597 & 0.0522 & 0.2644 & 2.6974 \\
\hline 2009 3st Quarter & 341.1397 & 7.0061 & 2.6670 & 0.3024 & 0.6441 & 2.5234 \\
\hline 2009 4st Quarter & 331.7963 & 5.8755 & 2.3986 & 0.0562 & 0.2311 & 1.4564 \\
\hline 2010 1st Quarter & 330.3032 & 5.7746 & 0.3119 & 0.1674 & 0.3149 & 2.2529 \\
\hline 2010 2st Quarter & 335.7331 & 5.9488 & 0.5356 & 0.1034 & 0.6976 & 2.2383 \\
\hline 2009 3st Quarter & 336.3403 & 5.9938 & 1.2822 & 0.0944 & 0.3883 & 2.4474 \\
\hline 2010 4st Quarter & 336.9384 & 6.1592 & 0.6164 & 0.0800 & 0.3465 & 2.2501 \\
\hline 2011 1st Quarter & 338.0949 & 6.2829 & 0.6090 & 0.0900 & 0.5828 & 2.8106 \\
\hline 2011 2st Quarter & 338.5210 & 6.3358 & 0.7012 & 0.0712 & 0.4720 & 2.0457 \\
\hline 2011 3st Quarter & 341.1914 & 6.3492 & 0.5046 & 0.0798 & 0.4689 & 2.0848 \\
\hline 2011 4st Quarter & 340.8621 & 6.2496 & 0.3977 & 0.0880 & 0.3824 & 2.0065 \\
\hline 2012 1st Quarter & 340.7435 & 5.9917 & 0.4485 & 0.0736 & 0.3449 & 1.5685 \\
\hline 2012 2st Quarter & 343.8052 & 6.3417 & 0.5008 & 0.0892 & 0.3192 & 1.8331 \\
\hline 2012 3st Quarter & 345.6949 & 6.5730 & 0.4417 & 0.0876 & 0.1943 & 1.7293 \\
\hline 2012 4st Quarter & 346.2501 & 6.7776 & 0.4682 & 0.0853 & 0.2196 & 1.6127 \\
\hline 2013 1st Quarter & 340.1915 & 6.3115 & 0.4437 & 0.0713 & 0.3061 & 0.0020 \\
\hline 2013 2st Quarter & 343.4145 & 6.3021 & 0.5919 & 0.0706 & 0.2293 & 1.5928 \\
\hline
\end{tabular}

Simulating the data through successive sweep method in Eviews 6.0 software, the co-evolution mode for dynamic performance evaluation on energy saving and emission

$$
\left\{\begin{array}{l}
\frac{d x_{1}}{d t}=1.162 x_{2}-1.127 \mathrm{x}_{3}+1.028 x_{5}-0.243 \mathrm{x}_{2}^{2}+0.249 x_{3}^{2}+0.047 x_{4}^{2}-0.117 x_{5}^{2}+0.043 x_{6}^{2}+f_{1}(t) \\
\frac{d x_{2}}{d t}=0.517+0.347 x_{1}+0.545 \mathrm{x}_{2}-1.541 x_{3}+0.505 x_{4}+0.807 x_{5}-0.757 x_{6}-0.078 x_{1}^{2}+0.342 x_{3}^{2}+0.205 x_{4}^{2}-0.109 x_{5}^{2}+0.045 x_{6}^{2}+f_{2}(t) \\
\frac{d x_{3}}{d t}=3.612 x_{1}-5.014 x_{2}+1.384 x_{4}+1.721 x_{5}-0.484 x_{6}+0.180 x_{1}^{2}+0.626 x_{2}^{2}-1.179 x_{4}^{2}-0.189 x_{5}^{2}-0.0187 x_{6}^{2}+f_{3}(t) \\
\frac{d x_{4}}{d t}=-1.164-1.155 x_{1}+4.914 x_{2}-3.379 x_{3}+3.353 x_{4}+0.18 x_{5}-1.935 x_{6}-0.564 x_{2}^{2}+0.79 x_{3}^{2}+0.085 x_{6}^{2}+f_{4}(t) \\
\frac{d x_{5}}{d t}=1.243-1.982 x_{1}+x_{3}+1.018 x_{5}+0.196 x_{6}+0.127 x_{1}^{2}-0.472 x_{3}^{2}-0.0193 x_{6}^{2}+f_{5}(t) \\
\frac{d x_{6}}{d t}=-2.25 x_{1}+2.886 x_{2}-0.884 x_{3}+1.382 x_{4}+1.019 x_{6}+0.242 x_{1}^{2}-0.622 \mathrm{x}_{2}^{2}-0.863 x_{4}^{2}-0.04 x_{5}^{2}+f_{5}(t)
\end{array}\right.
$$

The goodness of fit of each evolution equation is very high, which is shown in the following table:

By examining the residuals of each evolution equation, the residuals of each equation are satisfied normal with reduction of thermal power plant could be obtained as follows: mean 0 and variance 1 . Therefore, when analyzing the evolution of the plant's energy saving and emission reduction performance, the fluctuations a can be temporarily ignored. 
Table 2. The goodness of fit of each evolution equation.

\begin{tabular}{lllllll}
\hline Equation & X1 & X2 & X3 & X4 & X5 & X6 \\
\hline $\begin{array}{l}\text { Goodness of } \\
\text { fit }\left(\mathrm{R}^{2}\right)\end{array}$ & 0.991 & 0.995 & 0.911 & 0.944 & 0.932 & 0.882 \\
\hline
\end{tabular}

As can be seen from the results of evolutionary model, the damping coefficients of six state variables are $\quad a_{1}=0, a_{2}=0.545, a_{3}=0, a_{4}=3.353, a_{5}=1.018, a_{6}=1.019$

respectively. Seen from the above model, the damping coefficient stands for the change rate of variable. The greater the coefficient is, the faster the variable changes, so it is the fast variables. On the contrary, it is slow variable in the system, namely, the order parameter. In this model, the damping coefficients of coal consumption rate $\left({ }^{x_{1}}\right)$ and water consumption per unit power $\left(x_{3}\right)$ are zero, which are the smallest of the five variables. It indicates that the coal consumption rate and water consumption per unit power does not change, appear no damping phenomenon when the system is in critical state, which in line with the basic characteristics of the order parameter. Thus, the coal consumption and water consumption per unit power two variables are the order parameter of empirical analysis object during the study period.

The coal consumption rate and water consumption per unit power are key indicators reflecting the energy saving and emission reduction performance in thermal power plant. If the coal consumption rate is higher, it means that the unit operation efficiency is low. Since the level of coal consumption rate is related with coal quality, the higher coal consumption rate means a worse coal quality and a higher emission level of pollutants. Therefore, the coal consumption can reflect the energy saving and emission reduction performance. Water consumption per unit power is a key indicator of energy consumption in thermal power plant. Thus, The coal consumption rate and water consumption per unit power are two order parameters that play a key role in determining the evolution of energy saving and emission reduction in the thermal power plant. To realize the dynamic evaluation of energy saving and emission reduction performance, the research on the two variables is the key, which has great significance.

\section{Conclusion}

In this paper, the synergetic characteristics of energy saving and emission reduction in thermal power plant are analyzed to build a co-evolution model of dynamic evaluation. According to the empirical analysis results, the coal consumption rate and water consumption per unit power were the order parameters of energy saving and emission reduction in thermal power plant. The acquisition of order parameters provided some indicators sense for further study of the dynamic evaluation of thermal power enterprises energy saving and emission reduction performance.

\section{Acknowledgments}

This study is supported by the National Natural Science Foundation of China (Grant no. 71373076) and the Humanities and Social Science project of the Ministry of Education of China (Project no. 11YJA790217).The authors are grateful to the editor and anonymous reviewers for their suggestions on improving the quality of the paper.

\section{References}

[1] Chen. Shen, Meng-rui. Deng." Resource-oriented energy saving and emission reduction evaluation system of enterprise," Wuhan University of Technology, vol.32, no.4, pp. 49-52.2012.

[2] "Coal-fired power generation enterprises clean production evaluation guidelines," National Energy Board, 2012.

[3] "Thermal power industry clean production evaluation index system (pilot)," National Development and Reform Commission, 2012.

[4] Harken, Zhi-an. Guo, "Higher Synergetics," Beijing Science Press.1989.

[5] Liening Andreas. Strunk Guido. Mittelstaedt Ewald." Management Learning in Times of Crisis: An Experimental Study based on Synergetics," Nolinear dynamics psychology and life sciences, vol.17, no.4, pp.517-541.2013.

[6] Rajaram, Rajeev. Castellani, Brian. "Modeling complex systems macroscopically: Case agent based modeling, synergetics, and the continuity equation," Complexity, vol.18, no.2, pp.8-17.2012.

[7] Si-Feng Liu. Yao-guo Dang. Zhi-geng. Fang,” System theory and application," Beijing: Science Press,no.5, pp.146-147.2010.

[8] Shi-yong Li. et al," Nonlinear Science and Complexity Science," Harbin Institute of Technology Press, pp.44-53.2006.

[9] Qing-hong Zeng. et al (Translation)," From chaos to order," Shanghai Translation Publishing House. 1987.

[10] Ze-hai Song. "A Study on Integrated Mechanism of Metallurgy Enter Prise Technology Innovation Based on Synergetic Theory,” Tian Jin University. 2006.

[11] Jing-qi Sun. Dong-xiao Niu. Chun-jie Li." Empirical Study of the Complex Characteristics of the Electricity Market and its Evolution," Economic and Management Research, no.11,pp.3-8.2012.

[12] Xiao-bin Hong. Wang Han. Wang Tao. "Dynamic cooperative identification based on synergetics for pipe structural health monitoring with piezoceramic transducers," smart materials and structures, vol,22, no.4. 2013.

[13] Yao Yang. Technique evolutionary mechanisms and organizational order parameter [J]. Dialectics of Nature, 2007,23 (4) :47-49, 78. 
[14] Jing-qi. Sun. Dong-xiao. Niu. Chun-jie Li. " Recognition of the electricity market operator status based on complex systems theory," Management Science, vol.6,no.25, pp.111-118.2012.

[15] Wang Ping. Zhang Kuanyu. "The synergetic theory of enterprise management in the dynamic and complicated environment," Integration and innovation through measurement and management. pp. 2327-2334. 2006.
[16] Yin-peng. Chen." Energy saving and emission reduction performance evaluation based on osculating value method," Ecology and Environmental Sciences, vol.19, no.2, pp.419-422,2010.

[17] Dong Qing Xiao-yan Wang.” Thermal power plant energy evaluation index system," China Electric Power Education, no.9, pp. 56-57, 2011. 